\title{
Status of the CUORE experiment at Gran Sasso
}

\section{S. Di Domizio*i}

INFN - Sezione Di Genova, Via Dodecaneso 33, I-16146, Genova, Italy

E-mail: sergio.didomiziodge.infn.it

CUORE (Cryogenic Underground Observatory for Rare Events) is a second generation neutrinoless double beta decay experiment whose sensitivity is expected to be in the range of 41-95 meV for the electron neutrino effective mass. In CUORE the decay of $9.6 \cdot 10^{26}{ }^{130} \mathrm{Te}$ nuclei $(206 \mathrm{~kg})$ is observed by means of $988 \mathrm{TeO}_{2}$ crystals acting as bolometers at very low temperature $(\sim 10 \mathrm{mK})$. The experiment is located at the Laboratori Nazionali del Gran Sasso in Italy and is now approaching the final stage of construction. Most of the crystals have been produced, and the final test of the CUORE assembly line (called CUORE-0) is expected to be up and running by 2012. The construction of the full CUORE detector will begin in 2012 and is expected to finish by 2014 . The paper will summarize the physics reach of the experiment, the status of the construction, and the expected sensitivity.

36th International Conference on High Energy Physics,

July 4-11, 2012

Melbourne, Australia

\footnotetext{
* Speaker.

†n behalf of the CUORE collaboration
} 


\section{Introduction}

Our understanding of neutrino properties has been subject to great improvements in recent years. Lepton flavor non conservation is now considered a satisfactory explanation for the results of solar, atmospheric, reactor and accelerator neutrino oscillation experiments. We measured all the angles of the lepton mixing matrix, and we know that neutrinos have a non vanishing mass. However this picture is still incomplete. The value of the CP phases, as well as the neutrino mass hierarchy, are unknown. We do not know the absolute value of the neutrino mass scale, and whether it is a Dirac or a Majorana particle.

In contrast to the other properties, the absolute mass scale and the possible Majorana nature of neutrinos cannot be investigated by mean of oscillation experiments. In particular, the study of double beta decay [1] (DBD) is considered the only practical way of distinguishing between Dirac and Majorana neutrinos. This spontaneous (albeit very rare) process, in which a nucleus changes its atomic number by two units, can occur only in some nuclei with an even number of neutrons and protons, where the single beta decay is energetically forbidden. In two neutrino double beta decay (2vDBD) the process is accompanied by the emission of two electrons and two antineutrinos. This decay mode is allowed by the Standard Model of particle physics and has indeed been observed for all the interesting DBD isotopes, with half lives in the range $10^{19} \div 10^{21} \mathrm{y}$. There is also the possibility that only two electrons are emitted in the decay. This process is called neutrinoless double beta decay (0vDBD). It violates the lepton number conservation by two units and its existence would imply that the neutrino is a Majorana particle. Apart for a controversial claim in ${ }^{76} \mathrm{Ge}$ [2], which is now being scrutinized by other experiments, there is no evidence for this decay mode, and the half life lower limits are in the range $10^{22} \div 10^{25} \mathrm{y}$. The 0vDBD decay rate can be expressed as

$$
\Gamma_{0 v}=\frac{\ln 2}{T_{1 / 2}^{0 v}}=\ln 2 G^{0 v}\left|M^{0 v}\right|^{2} \frac{m_{\beta \beta}^{2}}{m_{e}^{2}}
$$

where $G^{0 v}$ is a phase space factor, $M^{0 v}$ is the matrix element for the nuclear transition, $m_{e}$ is the electron mass and $m_{\beta \beta}=\left|\sum_{i} U_{e i}^{2} m_{i}\right|$ is the effective neutrino Majorana mass. If the neutrino mixing angles and $\Delta m^{2}$ values measured by oscillation experiments are inserted in $m_{\beta \beta}$, it can be expressed as a function of the mass hierarchy, the mass of the lightest neutrino, and the Majorana $\mathrm{CP}$ phases. Therefore a measurement of 0vDBD half life could also give information on these unknown parameters. However, while the mere observation of 0vDBD would imply that the neutrino is a Majorana particle, extracting information about these others neutrino mass properties would require to know the matrix element for the nuclear transition with sufficient accuracy. Moreover the knowledge of the nuclear matrix elements is important for comparing the results of experiments carried out with different DBD isotopes.

From an experimental perspective, the two electrons emitted in 0vDBD produce a monochromatic peak in the energy spectrum, centered at the Q-value of the decay, which is in the $(2 \div 4) \mathrm{MeV}$ region, depending on the candidate isotope. The sensitivity of a 0vDBD experiment is defined as the half life corresponing to the minimum number of signal counts that can be observed over a given background, $S^{0 v}=\ln 2 N_{a} \frac{a}{A} \varepsilon \sqrt{\frac{M \cdot t}{b \cdot \Delta E}}$. In this formula $N_{a}$ is the Avogadro number, $a$ is the abundace of the DBD candidate nucleus, $A$ is the atomic mass number, $\varepsilon$ is the detection efficiency, $M$ 
is the detector mass, $t$ the measuring time, $b$ the background index expressed in counts/(keV $\cdot \mathrm{kg} \cdot \mathrm{y})$ and $\Delta E$ is the energy resolution. Given the low decay rate, the observation of the 0vDBD peak requires detectors with large source mass, excellent energy resolution and very low background. It is worth noting that $2 \mathrm{vDBD}$, which is a few orders of magnitude more intense than $0 \mathrm{vDBD}$, gives rise to a continuous spectrum whose end point coincides with the 0vDBD peak, thus representing a background that cannot be eliminated. For this reason, even for an hypothetical zero background experiment, the energy resolution is very important.

The use of large mass bolometers for the detection of 0vDBD is nowadays recognized as one of the most promising approaches in this kind of research. Bolometers, better described in Sec. 2, are cryogenic calorimeters that can measure the temperature rise induced by an energy release in an absorber crystal. Because the detection principles of these devices are based on thermal mechanisms, they feature an excellent energy resolution. The CUORE [3] (Cryogenic Underground Observatory for Rare Events) collaboration is working on the construction of an experiment that will use tellurium dioxide $\left(\mathrm{TeO}_{2}\right)$ bolometers for the search of neutrinoless double beta decay of the ${ }^{130} \mathrm{Te}$ isotope $\left(\mathrm{Q}_{\beta \beta}=2527.5 \mathrm{keV}\right)$. The detector will be a tightly packed array of $988 \mathrm{TeO}_{2}$ bolometers made with natural tellurium, with a total mass of $741 \mathrm{~kg}\left(206 \mathrm{~kg}\right.$ in $\left.{ }^{130} \mathrm{Te}\right)$.

In Sec. 2 the experimental features of large mass bolometers are outlined, while in Sec. 3 we present the result of the pilot experiment CUORICINO. In Sec. 4 we discuss the changes that have been introduced in order to improve the sensitivity of CUORE with respect to CUORICINO, and present the current status of CUORE-0, the first tower of CUORE. Finally, in Sec. 5 we will present CUORE and give an update about the status of the detector construction.

\section{Bolometric detectors for double beta decay}

In an simplified picture a bolometer can be sketched as an absorber material that is weakly coupled to a constant temperature heat sink and is provided with a sensor for the signal readout. At cryogenic temperatures the heat capacity $\mathrm{C}$ of the absorber crystal can be low enough that the energy released by a particle could give rise to a temperature increase $(\Delta T=E / C)$ that can be measured by the sensor. Because the detection is based on thermal principles, bolometers feature an excellent energy resolution, but, on the other side, their time response is intrinsically slow. Depending on the mass and material, a bolometer signal can have a time evolution that range from few $\mu s$ to few $s$. Moreover, because bolometric detectors can be made of a wide range of materials, it is possible to build a device that is at the same time the DBD source and detector. Compared to the case where the source and the detector are distinct, this approach has the advantage of maximizing the detection efficiency as well as not to spoil the energy resolution due to the DBD electrons energy that would not be detected because of self-absorption in the passive source material.

The CUORE absorbers are $\mathrm{TeO}_{2}$ crystals that are grown with natural abundance of ${ }^{130} \mathrm{Te}$ (34.2\%). Each crystal has a size of $5 \times 5 \times 5 \mathrm{~cm}^{3}$, a mass of $0.75 \mathrm{~kg}$ and a typical heat capacitance of $10^{-9} \mathrm{~J} / \mathrm{K}$ at $10 \mathrm{mK}$. The crystals are held by a copper structure by means of Teflon supports that also represent the weak thermal coupling to the heat bath. The sensors are neutron transmutation doped (NTD) germanium thermistors that are glued on each crystal by mean of 9 glue spots. The resistance of the NTDs has a steep dependence on the temperature, $R(T)=R_{0} \exp \left(\sqrt{T_{0} / T}\right)$, and it suddenly drops when an energy release takes place in the absorber. By polarizing the sensor with a 
constant current, the thermal pulse is converted into a voltage signal that can be fed into the preamplification stage of the readout chain. In typical working conditions the sensor has a resistance of $100 \mathrm{M} \Omega$ and an energy release of $1 \mathrm{MeV}$ induces a resistance variation of about $3 \mathrm{M} \Omega$. Taking into account the polarization current, this translates into a voltage drop across the sensor of about $100 \mu \mathrm{V}$ per $\mathrm{MeV}$ of released energy. The produced pulse has a duration of a few seconds and a bandwidth of about $12 \mathrm{~Hz}$.

\section{CUORICINO}

The feasibility of a ton scale bolometric experiment was demonstrated by CUORICINO [4, 5], a pilot experiment that took data at LNGS between 2003 and 2008. The CUORICINO detector was an array of $62 \mathrm{TeO}_{2}$ bolometers with a total mass of $41 \mathrm{~kg}\left(11 \mathrm{~kg}\right.$ in $\left.{ }^{130} \mathrm{Te}\right)$, maintained at about $10 \mathrm{mK}$ by a dilution refrigerator. The bolometers were arranged in a tower of 13 floors. Eleven floors were made of four $5 \times 5 \times 5 \mathrm{~cm}^{3}$ crystals with natural abundance of ${ }^{130} \mathrm{Te}$, while two floors were made of nine $3 \times 3 \times 6 \mathrm{~cm}^{3}$ crystals each. Two of these smaller crystals were enriched in ${ }^{128} \mathrm{Te}\left(82 \%\right.$ i.a.) and two in ${ }^{130} \mathrm{Te}(75 \%$ i.a.). The CUORICINO tower was surrounded by a $2 \mathrm{~cm}$ minimum thickness lead shield, maintained at $600 \mathrm{mK}$, to protect the bolometers from radioactive contaminations in the cryostat materials. Outside the cryostat, a $20 \mathrm{~cm}$ thick lead shield protected the apparatus from environmental gamma radioactivity in the underground laboratory. The external lead shield was surrounded by a borated polyethylene shield against neutrons, and the space between the gamma and neutron shield was maintained in nitrogen overpressure to reduce the concentration of radon in the proximity of the apparatus. Finally, the neutron shield was contained in a Faraday cage to reduce the noise from electromagnetic sources. The bolometers were calibrated monthly by inserting ${ }^{232} \mathrm{Th}$ sources in the proximitiy of the detectors. The energy resolution in the DBD region was on average $6 \mathrm{keV}$ FWHM and $10 \mathrm{keV}$ FWHM for the big and small crystals respectively, while the calibration residuals were less than one $\mathrm{keV}$ in the same energy region.

In more than five years of data taking CUORICINO collected a statistics of $19.8 \mathrm{~kg}\left({ }^{130} \mathrm{Te}\right) \cdot \mathrm{y}$. A background rate of 0.2 counts/(keV·kg.y) was measured around the DBD Q-value, and no evidence for a peak was found. CUORICINO set a 90\% C.L. lower limit on the 0vDBD half life of $T_{1 / 2}^{0 v}\left({ }^{130} \mathrm{Te}\right)>2.8 \times 10^{24} y$. This translates into an upper limit on the effective neutrino Majorana mass of $m_{\beta \beta}<(0.3 \div 0.7) \mathrm{eV}$, where the range is due to the different results of the theoretical calculation of the nuclear matrix elements taken into account.

\section{From CUORICINO to CUORE}

The goal of the CUORE experiment is to reach a sensitivity of a few $10^{26} \mathrm{y}$ on the 0vDBD half life of ${ }^{130} \mathrm{Te}$, about two order of magnitude better than CUORICINO. If this result will be achieved, CUORE will start exploring the inverted hierarchy region of the effective Majorana neutrino mass spectrum. The target sensitivity will be obtained mainly by increasing the detector mass, reducing the background and improving the energy resolution. While all the above aspects are important, the background abatement is the most challenging aspect from the scientific point of view. From a detailed study of the CUORICINO data and with the help of Monte Carlo simulations, it appeared that the dominant component of the background around the 0vDBD Q-value originates from surface 
alpha contaminations of the crystals and of the passive materials surrounding them. An intense R\&D activity was carried out in the past years to pinpoint the source of this background and to develop more effective material cleaning techniques. Dedicated test measurements performed with small setups indicate that most of the sufrace alpha contaminations come from the copper frames used to hold the crystals. Using the outcome of these tests as an input for a Monte Carlo simulation of the background, and taking into account a CUORE-like geometry, the copper contribution is a factor of four above the CUORE background goal of 0.01 counts $/(\mathrm{keV} \cdot \mathrm{kg} \cdot \mathrm{y})$, while the contribution from all other materials directly facing the bolometers is below this value.

CUORE-0, the first tower of CUORE, was built recently with the double purpose of performing a complete test of the CUORE detector assembly line, and having a high statistics check of the background reduction that is expected for CUORE. All the detector assembly procedure, including the materials handling and cleaning, was performed in the same way and with the same tools developed for CUORE. With approximately the same detector mass as CUORICINO, but a lower background, CUORE-0 will be also a competitive 0vDBD experiment by itself. The background, dominated by radioactive contaminations in the cryostat materials, is expected to be in the range $(0.05 \div 0.11)$ counts $/(\mathrm{keV} \cdot \mathrm{kg} \cdot \mathrm{y})$, and in two years of data taking the CUORE-0 sensitivity will be twice as the one of CUORICINO. The apparatus was cooled down recently in the former CUORICINO cryostat. All the 52 bolometers are working properly and are now undergoing the optimization that is needed before starting the data taking.

\section{Status of the CUORE experiment}

If the CUORE collaboration will succeed in achieving a background of 0.01 counts/(keV·kg $\cdot \mathrm{y})$ and an average energy resolution of $5 \mathrm{keV}$ FWHM around the 0vDBD Q-value, the experiment will reach in five years of data taking a $1-\sigma$ sensitivity of $1.6 \times 10^{26}$ years on the $0 \mathrm{vDBD}$ half life of ${ }^{130} \mathrm{Te}$. Taking into account several calculations of the nuclear matrix elements, this translate into an upper limit in the range $41-95 \mathrm{meV}$ on the effective Majorana neutrino mass, enough to start exploring the inverted hierarchy region of the nuetrino mass spectrum.

The 988 bolometers of CUORE will be arranged in 19 towers. Each tower, very similar to CUORICINO, will be composed by 52 crystals arranged in 13 floors. The bolometers will be kept at $10 \mathrm{mK}$ by a custom cryogen-free dilution refrigerator. They will be held by a suspension system mechanically decoupled from the cryostat, to reduce the amount of vibrations coming from the external environment and from the apparatus itself. The bolometer array will be shielded from environmental $\gamma_{s}$ and neutrons. An internal $6 \mathrm{~cm}$ minimum thickness lead shield, maintained at about $600 \mathrm{mK}$, will protect the bolometers from the radioactive contaminations of the cryostat itself. Externally, at room temperature, the cryostat will be surrounded by a further $25 \mathrm{~cm}$ minimum thickness lead shield and by a $18 \mathrm{~cm}$ polyethylene shield against neutrons. The space between the two external shields will be filled with $2 \mathrm{~cm}$ of $\mathrm{H}_{3} \mathrm{BO}_{3}$ powder. The cryostat will be contained in Faraday cage, and in a clean room provided with an anti-radon system.

The commissioning of the CUORE cryostat started in 2012 at LNGS and will be complete before the end of 2013. The dilution unit was delivered and tested, and 3 of the 6 cryostat vessels are now at LNGS. All the CUORE crystals [6,7] will be at LNGS by the end of 2012, and the last batch of NTD sensors will be delivered at the begining of 2013. The cleaning of all the copper 
parts needed for the assembly of the detectors will be completed by the end of 2013. The assembly of a single CUORE tower consists essentially of three steps, the gluing of the NTD sensors on the crystals, the stacking of the crystals in the copper structure, and the wire bonding for the electrical connections of the sensors. The assembly line, successfully tested with the mounting of CUORE- 0 , is now ready. The first CUORE tower will be mounted at the beginning of 2013 and the detector will be complete by spring 2014, when it will be inserted in the cryostat. The apparatus will be closed and cooled down in the second half of 2014 and data taking will start before the end of the year.

\section{Acknowledgments}

CUORE is supported by the Istituto Nazionale di Fisica Nucleare (INFN); the Director, Office of Science, of the U.S. Department of Energy under Contract Nos. DE-AC02-05CH11231 and DE-AC52-07NA 27344; the DOE Office of Nuclear Physics under Contract Nos. DE-FG0208ER41551 and DEFG03-00ER41138; the National Science Foundation under Grant Nos. NSFPHY-0605119, NSF-PHY-0500 337, NSF-PHY-0855314, and NSF-PHY-0902171; the Alfred P. Sloan Foundation; and the University of Wisconsin Foundation. The author is supported by INFN.

\section{References}

[1] S. R. Elliott, Mod. Phys. Lett. A 27 (2012) 1230009

[2] H. V. Klapdor-Kleingrothaus et al., Phys. Lett. B 586 (2004) 198

[3] C. Arnaboldi et al. [CUORE Collaboration], Nucl. Instrum. Meth. A 518 (2004) 775

[4] C. Arnablodi et al., Astropart. Phys. 34 (2011) 822

[5] S. Di Domizio [CUORICINO Collaboration], J. Phys. Conf. Ser. 375 (2012) 042014.

[6] C. Arnaboldi et al., Journal of Crystal Growth 312 (2010) 2999

[7] F. Alessandria et al., Astropart. Phys. 35 (2012) 839 